\title{
Sustitución valvular aórtica quirúrgica en pacientes de mediana edad. Prótesis mecánica versus biológica
}

\author{
Dra. María Victoria Ramos
}

\begin{abstract}
Resumen
Los pacientes de mediana edad que requieren sustitución valvular aórtica quirúrgica constituyen un grupo en el que la decisión del tipo de prótesis a implantar (biológica o mecánica) continúa siendo discutida. Se realizó una búsqueda bibliográfica con el objetivo de determinar cuál de las prótesis beneficia más a este grupo. Cuando se evalúan las bioprótesis, la principal dificultad radica en el deterioro estructural, más intenso cuanto más joven es el paciente y que puede requerir reintervención por disfunción severa. Por otro lado, las prótesis mecánicas implican anticoagulación de por vida, con los riesgos derivados de este tratamiento. En el presente artículo se analiza la evidencia más significativa para orientar al médico clínico en la toma de decisiones.
\end{abstract}

Palabras clave: VÁLVULA AÓRTICA-PRÓTESIS VALVULARES CARDÍACAS

BIOPRÓTESIS-EFECTOS ADVERSOS

PACIENTES-MEDIANA EDAD

\section{Surgical aortic valve replacement in middle-aged patients. Mechanical versus biological prosthesis}

\author{
Summary \\ process. \\ Key words: $\quad$ AORTIC VALVE-HEART VALVE PROSTHESIS \\ BIOPROSTHESIS-ADVERSE EFFECTS \\ PATIENTS-MIDDLE AGED
}

Middle-aged patients who require surgical aortic valve replacement constitute a group in wich the decision of the type of prosthesis to implant (biological or mechanical) continues to be discussed. A bibliographic search was carried out in order to determine which of the prostheses benefits this group. When evaluating bioprostheses, the main difficulty lies in structural deterioration, more intense in the younger patient and that may require reintervention due to severe dysfunction. On the other hand, the mechanical prosthesis involve anticoagulation for life, with the risks derived from this treatment. The present article analyzes the most significant evidence to guide the clinical doctor in decision making

\section{Introducción}

En las últimas décadas se ha asistido a un cambio en el uso de prótesis valvulares. Los nuevos modelos de prótesis biológicas con mayor durabilidad y las desventajas de la anticoagulación cuando se utilizan prótesis mecánicas han inclinado la balanza hacia la elección de bioprótesis. La Sociedad de Cirugía Cardíaca de Gran Bretaña e Irlanda reportó un aumento de $65 \%$ a $78 \%$ en el período comprendido entre 2004 y 2009 en el uso de prótesis biológicas ${ }^{(1)}$.
En las guías americanas de práctica clínica publicadas recientemente se considera razonable el implante de prótesis mecánica en pacientes menores de 50 años, siempre que no presenten contraindicación para recibir anticoagulantes, constituyendo indicación IIa, nivel de evidencia B.

En los pacientes mayores de 70 años, la bioprótesis se considera una opción razonable (IIa B). Por otra parte, en el grupo de pacientes de mediana edad (50-70 años) es razonable individualizar el tipo 
de válvula a implantar, aceptando que existen datos conflictivos en cuanto al beneficio en la sobrevida ${ }^{(2)}$. Por lo tanto, en estos pacientes, la elección continúa siendo problemática: en las bioprótesis, el deterioro estructural se establece en 10 a 15 años, pudiendo ser necesaria la reintervención, y en el caso de las prótesis mecánicas, la anticoagulación y el control estricto del INR son difíciles de alcanzar, generando morbilidad importante $\mathrm{e}^{(3)}$.

Con la finalidad de obtener evidencia actualizada sobre este tema se realizó una búsqueda bibliográfica en pubMed incluyendo las palabras clave: válvula aórtica, sustitución valvular, prótesis valvular mecánica y biológica, entre otras. Asimismo, se revisaron las referencias bibliográficas citadas en las guías de práctica clínica.

\section{Tipos de prótesis}

Las prótesis mecánicas en posición aórtica utilizadas inicialmente fueron las "ball and cage" en 1960 (Starr-Edwards). Posteriormente surgieron otras como las mono-disco y bi-disco. Dentro de las mono-disco, actualmente no disponibles, se citan las prótesis Bjork-Shiley y Medtronic-Hall. En la práctica estándar actual se utilizan las prótesis bi-disco. Dentro de ellas se incluyen: CarboMedics, Advancing the Standard Medical (ATS) y Medtronic. Sus componentes principales son carbono, teflón, dacrón, titanio y poliéster, lo que les confiere una larga duración.

La prótesis On-X fue introducida en Europa en 1996 y difiere de las demás en su composición (carbono pirolítico puro).

Dentro de las bioprótesis solo se mencionará que existen válvulas porcinas y de pericardio bovino (se describirán otros aspectos en detalle al referirnos al deterioro estructural). Dentro de las primeras se encuentran: Medtronic Mosaic, St. Jude Medical Biocor y Biocor Supra. Las prótesis de pericardio bovino incluyen Carpentier-Edwards Perimount, CE Perimount Magna, Sorin Soprano y CarboMedics Mitroflow ${ }^{(4)}$. Existe evidencia de que esta última presenta mayor deterioro estructu$\operatorname{ral}^{(5,6)}$.

\section{¿Cuál elegir?}

Múltiples estudios han intentado determinar qué prótesis es más adecuada para los pacientes según la edad, persistiendo el debate en los menores de 70 años con resultados a favor y en contra de las bioprótesis. Aquellos trabajos que apoyan su implante evalúan las técnicas de reemplazo endovascular co- mo una alternativa ventajosa cuando se produzca el deterioro estructural de la válvula implantada ${ }^{(3)}$.

Las guías realizan recomendaciones basadas en estudios randomizados que datan de principios de la década de 1990. Tras 20 años de desarrollo de nuevas prótesis y técnicas quirúrgicas es necesaria una revisión ${ }^{(7)}$. El estudio randomizado Veterans Affairs Cooperative reclutó 575 hombres entre 50 y 70 años; 394 se aleatorizaron para recibir prótesis biológica Hancock de primera generación versus prótesis mecánica Bjork-Shiley. En el seguimiento de 15 años la mortalidad fue significativamente mayor en el grupo de las bioprótesis $(79 \%$ versus $66 \%{ }^{(8)}$. El Edinburgh Randomized Trial comparó prótesis mecánica versus dos marcas de prótesis biológicas (Hancock y Carpentier-Edwards) en 533 pacientes de 54 años: el beneficio de la prótesis mecánica no se mantiene cuando el seguimiento se extiende a 20 años. Sin embargo, las prótesis utilizadas en ese entonces son actualmente obsoletas.

La escasez de estudios randomizados secundaria a las dificultades técnicas impulsó la realización de varios estudios observacionales. Lund y Bland publicaron un metaanálisis de 17.439 pacientes sometidos a sustitución valvular aórtica (SVA) con prótesis biológica versus mecánica ${ }^{(9)}$. Se incluyeron trabajos comprendidos entre 1989 y 2004 con la finalidad de determinar el impacto de los diferentes tipos de prótesis disponibles: mecánicas (mono-disco y bi-disco) y biológicas (pericardio porcino y bovino). Las diferencias en mortalidad cuando se comparan ambos tipos de prótesis desaparecen cuando se corrige por edad y por factores de riesgo conocidos como cirugía de revascularización miocárdica y clase funcional de la New York Heart Association (NYHA) III o IV.

Utilizando los modelos contemporáneos de prótesis varios estudios han señalado una tendencia a menor mortalidad con las prótesis mecánicas, llegando a establecer el punto de corte en 65 años ${ }^{(10)}$. Algunos autores señalan que muchos de los estudios presentan defectos metodológicos por incluir pacientes de mayor edad y diversos tipos de bioprótesis que actúan como factores de confusión a la hora de obtener conclusiones ${ }^{(11)}$. Glaser y colaboradores publican en 2015 un estudio observacional de 4.545 pacientes de 50 a 69 años sometidos a SVA en el período comprendido entre 1997 y 2013 . La sobrevida de los pacientes que recibieron prótesis mecánica fue significativamente mayor comparada con la del grupo de bioprótesis ${ }^{(12)}$. Contrariamente, Marc Ruel y colaboradores, en un estudio retrospectivo de 314 pacientes menores de 60 años, sometidos a SVA aislada, no observaron diferencias significativas en la sobrevida en un seguimiento a 20 años ${ }^{(13)}$. 
Steven Khan, también durante 20 años de seguimiento de 1.389 pacientes menores de 65 años sometidos a SVA, obtuvo similares resultados ${ }^{(14)}$.

En el trabajo de Andrew Goldstone y colaboradores se evaluó la mortalidad de pacientes sometidos a SVA (biológica versus mecánica) estratificados en categorías de edad: 45 a 54 años y 55 a 64 años en el período 1996-2013. La mortalidad a 15 años fue significativamente mayor en los pacientes que recibieron bioprótesis, pero solo en el grupo de 45-54 años (30,6\% prótesis biológica vs $26,4 \%$ prótesis mecánica, HR $1,23 ;$ IC95\% $1,02-1,48 ; p=0,03)^{(15)}$.

\section{El problema del deterioro estructural}

El uso de válvulas biológicas data de inicios de 1960 y a lo largo del tiempo han sufrido muchas modificaciones.

Los autoinjertos utilizan la válvula pulmonar del propio paciente para implantarla en posición aórtica (procedimiento de Ross) con excelente perfil hemodinámico y baja tasa de complicaciones (degeneración, trombosis y endocarditis). Son adecuados en niños y adolescentes porque acompañan el crecimiento de la raíz aórtica.

Los homoinjertos son válvulas humanas criopreservadas que podrían ser útiles en casos de endocarditis extensa, pero no están ampliamente disponibles y tienden frecuentemente a la degeneración ${ }^{(16)}$.

Los más usados actualmente son los xenoinjertos, provenientes de tejidos animales, entre ellos las válvulas aórticas porcinas y de pericardio bovino. Las válvulas porcinas se fijan en un stent metálico, habitualmente de níquel-cobalto, mientras que las de pericardio bovino se diseñan imitando la válvula tricúspide. Los tejidos son sometidos a una serie de procedimientos preimplante que incluyen criopreservación en nitrógeno líquido, fijación en glutaraldehído, fijación a alta presión, y tratamiento antimineralización para prevenir la acumulación de calcio $^{(17)}$

El deterioro estructural es un proceso multifactorial caracterizado por deterioro progresivo de las valvas que trae como consecuencia la disfunción valvular (estenosis y/o regurgitación). Se consideran dos estadios: a) deterioro subclínico y b) deterioro clínicamente relevante. La definición de deterioro estructural subclínico se basa en criterios ecocardiográficos: 1) incremento en el gradiente medio transvalvular $>10 \mathrm{mmHg}$ con descenso concomitante en el área efectiva $>0,3 \mathrm{~cm}^{2}$ (y/o descenso en el índice de velocidad Doppler $>0,08$ ), y/o nueva regurgitación al menos leve o incremento de al menos 1 grado de una regurgitación intraprotésica pree- xistente, y 2) cambios en la morfología (engrosamiento, calcificación, flail, pannus) y/o en la movilidad de las valvas comparadas con el ecocardiograma basal posoperatorio(18).

Existe deterioro estructural clínicamente relevante cuando el gradiente medio transvalvular es $>20 \mathrm{mmHg}$ con descenso concomitante en el área efectiva $>0,6 \mathrm{~cm}^{2}$ (y/o descenso en el índice de velocidad Doppler $>0,15$ ), resultando en estenosis aórtica severa y/o nueva ocurrencia o incremento de insuficiencia intraprotésica de grado moderado a severo $^{(18)}$.

La patogenia del deterioro estructural valvular se ha atribuido al uso de glutaraldehído, tratamiento que procura la estabilización del tejido biológico y la disminución de la inmunogenicidad. Como contrapartida produce daño de membrana con influjo de calcio que se combina con fosfolípidos y produce cristales de calcio. Factores derivados del paciente y el estrés mecánico contribuyen al crecimiento de los $\operatorname{mismos}^{(19)}$.

En concordancia con el planteo de que el deterioro de la prótesis puede estar vinculado a un proceso inflamatorio similar al aterosclerótico, se ha visto que los niveles de proteína $\mathrm{C}$ reactiva están incrementados 3,7 veces en comparación con las válvulas nativas $^{(20)}$. La degeneración de la bioprótesis es un proceso mediado por el sistema inmune (celular y humoral), más agresivo en el paciente joven (20\% a $30 \%$ en pacientes menores de 40 años) ${ }^{(19)}$. Puede existir infiltrado de células mononucleares y macrófagos. Del mismo modo, anticuerpos IgG e IgM invaden la matriz valvular. Existe rotura de las fibras colágenas y calcificación ${ }^{(17)}$. Los depósitos de calcio se localizan habitualmente en las cúspides (calcificación intrínseca), aunque puede existir calcificación extrínseca, responsable del desarrollo de trombos o vegetaciones ${ }^{(21)}$. Antiguamente se creía que la calcificación era un proceso pasivo, no regulado y degenerativo. En la actualidad se considera que el mecanismo de depósito de calcio podría ser similar a la mineralización fisiológica del hueso y otros tejidos.

Si el deterioro estructural puede tener mecanismos compartidos con el proceso aterosclerótico, surge la interrogante del eventual beneficio del tratamiento con estatinas. En el mayor estudio ( $\mathrm{n}=$ 1.193), realizado por Kulik y colaboradores, no se pudo comprobar relación entre la terapia hipolipemiante, la progresión de gradientes medio y máximo, y la degeneración valvular estructural en un período de seguimiento de 1, 5 y 10 años ${ }^{(22)}$. Se ha propuesto que la ausencia de resultados positivos podría deberse a que el descenso de lípidos aislados no implica una mejoría en la longevidad de la prótesis 
porque existen otros mecanismos involucrados. Considerando las acciones pleiotrópicas de las estatinas, los efectos beneficiosos son independientes del nivel de lípidos, pero se argumenta que dichos beneficios a nivel bioquímico no se traducen clínicamente $^{(23)}$.

Otro aspecto no siempre tenido en cuenta es su efecto anticoagulante, que puede incrementar el sangrado perioperatorio. Las estatinas pueden generar una regulación en menos de la cascada de la coagulación, lo que se ha vinculado al descenso en la expresión del factor tisular (glicoproteína transmembrana implicada en la vía extrínseca de la coagulación). Existe reducción de la producción de trombina y consecuentemente las reacciones catalizadas por esta enzima. Por otro lado, las estatinas incrementan la expresión de trombomodulina en las células endoteliales potenciando la actividad de la proteína $\mathrm{C}$ anticoagulante ${ }^{(24)}$.

Las estrategias genéricas que se han propuesto para prevenir la calcificación incluyen: 1) terapia sistémica con agentes anticalcificación (bifosfonatos), 2) terapia local con dispositivos liberadores de droga y 3) modificación de los biomateriales que componen las prótesis ${ }^{(21)}$. Sin embargo, se requieren más investigaciones que validen su eficacia y seguridad.

La falla estructural comienza a partir de los ocho años y se incrementa enormemente luego de los diez años teniendo a partir de este momento efecto en la sobrevida ${ }^{(25)}$. La edad del paciente es el determinante de mayor importancia en el deterioro estructural de la prótesis, aunque también se ha vinculado a la presencia de hiperparatiroidismo e insuficiencia renal(19). Recientemente, la existencia de trombosis subclínica de las bioprótesis ha cobrado interés como factor contribuyente del deterioro estructural $^{(26)}$. En algunos estudios el hallazgo de motilidad reducida de los velos valvulares mediante tomografía axial computarizada o ecocardiograma transesofágico se ha atribuido a la presencia de trombosis subclínica, si bien aún no existe confirmación patológica ${ }^{(26)}$. Los pacientes con este fenómeno pueden presentarse con incremento significativo de los gradientes transvalvulares. La anticoagulación con warfarina constituye actualmente el único tratamiento que mejora la disminución de la movilidad de los velos en estudios observacionales, aunque algunos registros han mostrado resultados similares con el uso de anticoagulantes directos ${ }^{(27)}$.

En una situación de deterioro estructural, cuando existen parámetros de disfunción grave, puede ser necesaria la reintervención. En un estudio de seguimiento a diez años, que incluyó 672 pacientes (edad media: $72 \pm 8$ años) operados de SVA con bio- prótesis, 6,6\% tuvo degeneración valvular clínicamente relevante, requiriendo reintervención $83 \%$ de ellos; el deterioro estructural fue subclínico en $30,1 \%{ }^{(18)}$.

En aquellos pacientes con deterioro estructural clínico el reemplazo de la bioprótesis patológica usualmente mejora los síntomas y la hemodinamia. Sin embargo, existe controversia respecto al riesgo de estos pacientes, considerando que aumenta la mortalidad cuando se la compara con la primera cirugía. Se ha documentado una mortalidad de entre $3 \%-7 \%$ en varias series, dependiendo de los factores de riesgo y estatus del paciente ${ }^{(28)}$.

En un estudio retrospectivo de 3.356 pacientes se analizaron mortalidad y predictores de resultado adverso por falla de la bioprótesis aórtica previamente implantada. Del total de procedimientos, 1.338 fueron realizados con cirugía de revascularización miocárdica (CRM) concomitante y 2.142 consistieron solo en SVA. En el período comprendido entre 1975-1999, 312 pacientes requirieron reemplazo de la bioprótesis por deterioro estructural, con una mortalidad global de 6,8\%. En los pacientes con cirugía combinada (SVA y CRM) la mortalidad alcanzó 8,3\% y fue $6,6 \%$ en los casos de SVA aislada. Dentro de los predictores de riesgo, solo la clase funcional III de NYHA fue significativa ${ }^{(29)}$.

El uso de técnicas de implante transcatéter actualmente aprobadas para los pacientes de alto y moderado riesgo quirúrgico ha abierto el horizonte para aquellos que necesitan recambio protésico(30). Si bien aún no existen estudios con la potencia estadística suficiente para evaluar la no inferioridad con respecto a la cirugía, algunos trabajos han observado que el empleo de implante valvular aórtico transcatéter (TAVI) en el recambio protésico es una alternativa segura y eficaz a corto plazo en pacientes de alto riesgo ${ }^{(31,32)}$. En el registro multinacional valve-in-valve se incluyeron 459 pacientes con bioprótesis con deterioro estructural. La sobrevida a un año fue $83,2 \%$. Los factores asociados con mortalidad incluyeron: prótesis de menor tamaño $(\leq 21$ $\mathrm{mm}$ ) y grado de estenosis basal ${ }^{(33)}$.

\section{El problema de la anticoagulación}

Considerando lo previamente expuesto con respecto al proceso de deterioro de las prótesis biológicas y a la eventual necesidad de reintervención, la balanza se inclinaría hacia el uso de prótesis mecánicas. Sin embargo, su principal inconveniente es la obligatoriedad de anticoagulación para evitar la trombosis de la prótesis y el tromboembolismo. Se estima que el riesgo de trombosis protésica es cercano a $0,2 \%$ por año, siendo máximo en los primeros meses 
luego de la cirugía, debido a que los componentes del anillo no están completamente endotelizados. La incidencia estimada de eventos se sitúa entre $0,8 \%$ a $2,3 \%$ por paciente y año ${ }^{(4)}$. En un estudio retrospectivo que incluyó 2.982 pacientes sometidos a SVA con prótesis mecánica, ocurrieron 421 eventos tromboembólicos, de los cuales $46 \%$ fue accidente isquémico transitorio (AIT), $42 \%$ accidente cerebrovascular (ACV) y $10 \%$ embolia periférica ${ }^{(34)}$.

La warfarina ha tenido los mejores resultados en prevenir la incidencia de trombosis y tromboembolismo $^{(25)}$. Las dificultades de esta terapia incluyen los controles de INR de por vida y la complejidad para mantener valores en rango adecuado de anticoagulación.

El sangrado es otra complicación de la terapia anticoagulante. Algunos estudios apuntan al hecho de que $40 \%$ de los episodios de sangrado se produce en el primer año luego de la cirugía, cuando es más probable que haya importante fluctuación en los niveles de $\mathrm{INR}^{(4)}$. Las localizaciones más frecuentes de sangrado son el tracto digestivo y el sistema nervioso central. La incidencia de sangrado menor puede alcanzar el 10,5\% anual ${ }^{(25)}$. El riesgo de sangrado mayor, fundamentalmente intracraneano, se ha vinculado fuertemente a la edad y se estima en $2 \%-4 \%$ anual (versus $1 \%$ en pacientes con bioprótesis) ${ }^{(35)}$. Se ha constatado que en pacientes con prótesis mecánica e igual nivel de anticoagulación, a los siete años, las tasas de sangrado son siete veces mayores en pacientes de más de 60 años ${ }^{(25)}$.

Otro aspecto muy relevante a considerar es el riesgo de recurrencia del sangrado intracraneano. La información es limitada, pero de los datos disponibles en pacientes sin prótesis valvular que sufren hemorragia intracraneana espontánea, el riesgo de recurrencia es $2 \%-3 \%$ por año sin terapia antiagregante $o$ anticoagulante. En casos de hemorragia lobar que comprometa la corteza existe mayor riesgo de recurrencia comparados con hemorragias hemisféricas profundas. Cuando el paciente recibe aspirina, el riesgo de recurrencia alcanza $40 \%$ y se triplica con warfarina ${ }^{(36)}$.

A pesar de que los pacientes añosos son los más expuestos a presentar accidentes por anticoagulación, gran número de trabajos se han dedicado a evaluar su impacto en el grupo de mediana edad. Un estudio retrospectivo que incluyó a pacientes menores de 65 años sometidos a SVA con prótesis biológica versus mecánica mostró $7 \%$ de incidencia de complicaciones neurológicas (isquémicas y hemorrágicas) tardías vinculadas al uso de anticoagulantes, todas en el grupo que recibió prótesis mecánica(37). En dicho trabajo las contraindicaciones médicas y sociales para recibir anticoagulantes fueron: even- tos neurológicos previos, insuficiente adherencia al tratamiento, trastornos de la coagulación, mujer joven en edad reproductiva y negativa a recibir transfusiones. Por último, los autores consideran que los pacientes de 50-65 años que llevan un estilo de vida activo no son buenos candidatos para recibir prótesis mecánicas que requieren anticoagulación a largo plazo, siendo esta responsable de una incidencia significativamente alta de injuria sobre el sistema nervioso central tanto hemorrágica como tromboembólica.

Otros aspectos menos discutidos en la literatura son las extracciones frecuentes de sangre para control de INR, las interacciones farmacológicas y las restricciones dietéticas ${ }^{(38)}$. A ello se suma que la anticoagulación se discontinúa en caso de sangrado (inclusive en los banales) o frente a algunos procedimientos quirúrgicos, lo que predispone a tromboembolismo y trombosis de la prótesis que requiere cirugía de urgencia.

La introducción de los nuevos anticoagulantes orales para el tratamiento de la fibrilación auricular y su perfil de eficacia y seguridad alentaron la posibilidad de su uso en el caso de prótesis mecánicas como alternativa a la warfarina. Sin embargo, los resultados negativos del estudio en fase II RE-ALIGN han puesto un freno a investigaciones posteriores en este campo particular. El estudio RE-ALIGN fue un ensayo multicéntrico, randomizado y prospectivo que comparó warfarina versus dabigatrán en pacientes portadores de prótesis mecánicas en posición mitral y aórtica. El estudio fue interrumpido al constatar tasas inaceptables de eventos tromboembólicos y sangrados en el grupo tratado con dabigatrán ${ }^{(39)}$.

Dado que la warfarina tiene un lugar destacado en la anticoagulación de prótesis mecánicas deben mencionarse los resultados del estudio PROACT, que evaluó eficacia y seguridad de dosis menores de warfarina en pacientes portadores de prótesis mecánica On-X en posición aórtica. PROACT (prospectivo, randomizado y multicéntrico) demostró un descenso estadísticamente significativo en sangrados mayores y menores sin diferencias en la incidencia de AIT, ACV, eventos neurológicos totales ni mortalidad por todas las causas. Este estudio condujo a la aprobación por la Federal Drug Administration del uso de warfarina con INR objetivo de 1,5 a 2 y ácido acetilsalicílico $81 \mathrm{mg} /$ día en portadores de prótesis aórtica $\mathrm{On}-\mathrm{X}^{(40)}$.

\section{Válvulas mecánicas y su impacto en la calidad de vida}

Un aspecto que pocas veces es tomado en cuenta y 


\section{Resumen conceptual}

- La evidencia es conflictiva en cuanto a la elección de prótesis valvular aórtica en los pacientes de mediana edad.

- Algunos estudios han mostrado mejor sobrevida con el uso de prótesis mecánicas.

- El deterioro estructural constituye uno de los problemas principales del uso de prótesis biológicas; este puede ser subclínico o clínico.

- La patogenia del deterioro estructural presenta similitudes al proceso aterosclerótico, sin embargo no se ha demostrado mejora significativa con el tratamiento con estatinas.

- La trombosis subclínica se ha relacionado en estudios recientes con el deterioro estructural de las bioprótesis.

- La terapia anticoagulante es necesaria en los pacientes con prótesis mecánicas y solo la warfarina ha mostrado beneficios en prevenir la trombosis valvular.

- El uso de anticoagulantes en la mujer embarazada requiere un protocolo de cambio a heparina de bajo peso molecular para prevenir complicaciones fetales.

últimamente ha cobrado interés, es la repercusión negativa de la prótesis mecánica en la calidad de vida del paciente. Al considerar evitar una reintervención en los pacientes de menos edad, se olvidan frecuentemente los aspectos que afectan el día a día del individuo. Por un lado, la anticoagulación es de por vida y la predisposición al sangrado favorecida por estos fármacos puede eventualmente hacer necesario el uso de protectores para desempeñar actividades de la vida diaria o modificar el atuendo para no exponer áreas de equimosis ${ }^{(41)}$.

Por otro lado, la presencia permanente de un click audible puede constituirse en un problema ${ }^{(41)}$. Cuestionarios sobre calidad de vida han señalado este aspecto como uno de los más relevantes. El click audible puede ser causa de insomnio, interferir en la vida sexual y en casos extremos producir síndrome de estrés postraumático ${ }^{(41)}$.

\section{Elección de prótesis en la mujer}

A todos los aspectos previamente reseñados de la elección de prótesis en menores de 70 años se agrega la difícil situación de la mujer en edad fértil. Actualmente no existe un tipo óptimo de prótesis para esta situación, ya que cualquiera de ellas eleva el riesgo de insuficiencia cardíaca, arritmias o endocarditis durante el embarazo.
Las bioprótesis tienen más deterioro estructural debido a los cambios hemodinámicos de la gestación y se recomienda que la mujer con deseos de embarazo, realice la planificación familiar en los primeros cinco años luego del implante (aunque se han visto casos de deterioro manifiesto durante el embarazo o precozmente en el posparto) ${ }^{(16)}$. Como contrapartida, las pacientes con válvula mecánica tienen la más alta tasa de complicaciones materno-fetales. Los abortos espontáneos con warfarina y derivados alcanzan el $70 \%$, que desciende a $20 \%$ cuando se realiza la sustitución por heparina de bajo peso molecular. Las complicaciones cardíacas maternas son cercanas a $20 \%$ en las portadoras de prótesis mecánicas ${ }^{(16)}$ e incluyen trombosis de la válvula, tromboembolismo y sangrado. Estos datos tienen gran variabilidad debido a la ausencia de ensayos clínicos randomizados ${ }^{(43)}$.

El estado hipercoagulable del embarazo incrementa el riesgo de formación de trombos. Aunque se recomienda que la anticoagulación se realice con heparina de bajo peso molecular, el riesgo de tromboembolia es mayor que cuando se utiliza warfarina.

Por su parte, la heparina no fraccionada que tiene un mejor perfil de seguridad para el feto por no atravesar la placenta, tiene un alto riesgo de generar complicaciones tromboembólicas en la madre. Se reporta una incidencia de trombosis de 3,9\% en pacientes que toman warfarina sola, $9,2 \%$ en las que reciben heparina no fraccionada en el primer trimestre seguida de warfarina y $33 \%$ cuando solo se utiliza heparina no fraccionada ${ }^{(43,44)}$. Es oportuno destacar que los datos fueron obtenidos a partir de una revisión de Chan y colaboradores, donde $48 \%$ de las prótesis implantadas eran modelo cage and ball Starr-Edwards (actualmente en desuso por su mayor trombogenicidad).

La warfarina, que presenta el mayor beneficio para la madre, atraviesa libremente la placenta e incrementa sustancialmente la presencia de anomalías congénitas en el feto, especialmente en el primer trimestre. Específicamente en las semanas 6 a 12 puede generar una embriopatía que afecta a cartílago y hueso (condromalacia punctata), cuya incidencia se ha estimado en 5\%-10\%. Luego del primer trimestre se incrementa el riesgo de defectos a nivel del sistema nervioso central, presumiblemente por microhemorragias. El efecto podría ser dosis dependiente, con un punto de corte en $5 \mathrm{mg} /$ día $^{(45)}$.

Cuando es necesario el uso de anticoagulantes, existe un riesgo específico de sangrado de la placenta durante la gestación o en el parto ${ }^{(44)}$. En el estudio realizado por McLintock y colaboradores ${ }^{(44)}$, de pacientes embarazadas portadoras de prótesis me- 
cánicas tanto aórticas como mitrales, el sangrado prenatal estuvo presente en $17 \%$ de las pacientes y la mayoría fue de origen obstétrico. Hubo sangrado posparto en $19 \%$ y se vinculó al tratamiento con enoxaparina debido a la intensidad de dosis requerida para prevenir la trombosis y a la asociación con ácido acetilsalicílico. A pesar de este hecho, se recomienda mantener ambos fármacos para reducir el riesgo de trombosis.

\section{Conclusiones}

La elección del tipo de prótesis valvular en pacientes menores de 70 años debe realizarse evaluando cuidadosamente el riesgo asociado con la reintervención, la conveniencia del tratamiento anticoagulante, el estilo de vida y las preferencias del paciente. En la revisión realizada por Chikwe ${ }^{(35)}$, la elección de una prótesis biológica o mecánica es razonable para los pacientes de edad media sin un punto de corte absoluto.

Pese a la tendencia mundial a emplear prótesis biológicas en pacientes jóvenes, algunos expertos consideran que la evidencia disponible es insuficiente $^{(46)}$. Todos los especialistas involucrados deberían guiar al paciente conociendo los aspectos previamente reseñados y considerando que actualmente la prótesis ideal no existe.

\section{Agradecimiento}

Agradezco especialmente al Dr. Juan Carlos Reinoso por su colaboración con la idea original.

\section{Bibliografía}

1. Dunning J, Gao H,Chambers J, Moat $\mathbf{N}$, Murphy G, Pagano D, et al. Aortic valve surgery: Marked increase in volume and significant decreases in mechanical valve use-an analysis of $41,227 \mathrm{pa}-$ tients over 5 years from the Society for Cardiothoracic Surgery in Great Britain and Ireland National database. J Thorac Cardiovasc Surg 2011;142:776-82. doi:10.1016/j.jtcvs.2011.04.048.

2. Nishimura RA, Otto CM, Bonow RO, Carabello BA, Erwin JP, Fleisher LA, et al. 2017 AHA/ACC focused update of the $2014 \mathrm{AHA} / \mathrm{ACC}$ guideline for the management of patients with valvular heart disease:A Report of the American College of Cardiology/American Heart Association Task Force on Clinical Practice Guidelines. Circulation 2017;135(25): e1159-e1195. doi: 10.1161/CIR.0000000000000503.

3. BadhwarV, Ofenloch JC, Rovin JD, van Gelder HM, Jacobs JP. Noninferiority of closely monitored mechanical valves to bioprostheses overshadowed by early mortality benefit in younger patients.
Ann Thorac Surg 2012;93:748-53. doi: 10.1016/j.athorasurg.2011.12.032

4. Susak S, Velicki L, Popovic D, Burazor I. Surgical valve replacement (bioprosthetic vs. mechanical).En: Aikawa E, ed. Calcific Aortic Valve Disease. London: Intech Open; 2013. 361-78. doi: 10.5772/53687.

5. Sénage T, Le Tourneau T, Foucher Y, Pattier S, Cueff C, Michel M, et al. Early structural valve deterioration of Mitroflow aortic bioprosthesis. Mode, incidence, and impact on outcome in a large cohort of patients. Circulation 2014;130(23): 2012-20. doi:10.1161/CIRCULATIONAHA.114.010400.

6. Díaz R, Hernández-Vaquero D, Silva J, Pascual I, de la Hera JM, León V, et al. La degeneración real de la prótesis aórtica Mitroflow: análisis con riesgos competitivos. Rev Esp Cardiol 2017;70(12): 1074-81. doi: 10.1016/j.recesp.2017.01.035.

7. Suri RM, Schaff HV. Selection of aortic valve prostheses: contemporary reappraisal of mechanical versus biologic valve substitutes. Circulation 2013;128(12):1372-80. doi: 10.1161/CIRCULATION AHA.113.001681.

8. Hammermeister K, Sethi GK, Henderson WG, Grover FL, Oprian C, Rahimtoola SH. Outcomes 15 years after valve replacement with a mechanical versus a bioprosthetic valve: final report of the Veterans Affairs Randomized Trial. J Am Coll Cardiol 2000;36(4):1152-58. doi:10.1016/S0735-1097(00)00834-2.

9. Lund O, Bland M. Risk corrected impact of mechanical versus bioprosthetic valves on long term mortality after aortic valve replacement. J Thorac Cardiovasc Surg 2006; 132(1):20-6. doi: $10.1016 /$ j.jtcvs.2006.01.043.

10. Peterseim DS, Cen YY, Cheruvu S, Landolfo K, Bashore TM, Lowe JE, et al. Long-term outcome after biologic versus mechanical aortic valve replacement in 841 patients. J Thorac Cardiovasc Surg 1999;117(5):890-7. doi: 10.1016/S0022-5223(99)70368-5.

11. Weber A, Noureddinne H, Englberger L, Dick F, Gahl B, Aymard T, et al. Ten-year comparison of pericardial tissue valve versus mechanical prostheses for aortic valve replacement in patients younger than 60 years of age. J Thorac Cardiovasc Surg 2012;144(5): 1075-83. doi: 10.1016/j.jtcvs.2012.01.024.

12. Glaser N, Jackson V, Holzmann MJ, Franco-Cereceda A, Sartipy U. Aortic valve replacement with mechanical vs. biological prostheses in patients aged 50-69 years. Eur Heart J 2016; 37(34):2658-67. Download from http://eurheartj.oxfordjournals.org/. doi: 10.1093/eurheart/ehv580.

13. RuelM,Chan V, Bédard P, Kulik A, Ressler L, Lam BK, et al. Very long-term survival implications of heart valve replacement with tissue versus mechanical prostheses in adults $<60$ years of age. Circula- 
tion 2007;116(11 Suppl):I294-300.

doi: 10.1161/CIRCULATIONAHA.106.681429.

14. Khan SS, Trento A, DeRobertis M, Kass RM, Sandhu M, Czer LSC, et al. Twenty-year comparison of tissue and mechanical valve replacement. J Thorac Cardiovasc Surg 2001;122(2):257-69. doi: 10.1067/mtc.2001.115238.

15. Goldstone AB, Chiu P, Baiocchi $M$, Lingala $B$, Patrick WL, Fischbein MP, et al. Mechanical or biologic prostheses for aortic-valve and mitral-valve replacement. N Engl J Med 2017;377(19):1847-57. doi: 10.1056/NEJMoa1613792.

16. Hoffmann G, Lutter G, Cremer J. Durability of bioprosthetic cardiac valves. Dtsch Arztebl Int 2008;105(8):143-8. doi: 10.3238/arztebl.2008.0143.

17. Singhal P, Luk A, Butany J. Bioprosthetic heart valves: impact of implantation on biomaterials. Int Sch Res Notices Biomaterials 2013;2013:14p. doi: $10.5402 / 2013 / 728791$

18. Rodríguez-Gabella T, Voisine $\mathbf{P}$, Dagenais $\mathbf{F}$, Mohammadi S, Perron J, Dumont E, et al. Long-term outcomes following surgical aortic bioprosthesis implantation. J Am Coll Cardiol 2018; 71(13):1401-12. doi: 10.1016/j.jacc.2018.01.059.

19. Pibarot P, Dumesnil JG. Prosthetic heart valves. Selection of the optimal prosthesis and long-term management. Circulation 2009;119(7):1034-48. doi: 10.1161/CIRCULATIONAHA.108.778886.

20. Gilmanov D, Bevilacqua S, Mazzone A, Glauber M. Do statins low the process of calcification of aortic tissue valves? Interact Cardiovasc and Thorac Surg 2010;11(3):297-302. doi: 10.1510/icvts.2009.230920.

21. Schoen FJ, Levy RJ. Calcification of tissue heart valve substitutes: progress toward understanding and prevention. Ann Thorac Surg 2005;79(3): 1072-80. doi: 10.1016/j.athoracsur.2004.06.033.

22. Kulik A, Masters RG, Bédard P, Hendry PJ, Lam BK, Rubens FD, et al. Postoperative lipid-lowering therapy and bioprosthesis structural valve deterioration: justification for a randomised trial? Eur J Cardiothorac Surg 2010;37(1):139-44. doi: 10.1016/j.ejcts.2009.06.051.

23. Chacko J, Harling L, Ashrafian H, Athanasiou T. Can statins improve outcomes after isolated cardiac valve surgery? A systematic literature review. Clin Cardiol 2013;36(8):448-55 doi: 10.1002/clc.22140.

24. Undas A, Brummel-Ziedins KE, Mann KG. Statins and blood coagulation. Arterioscler Thromb Vasc Biol 2005;25(2):287-94. doi: 10.1161/01.ATV.0000151647.14923.ec.

25. Rahimtoola SH. Choice of prosthetic heart valve in adults: an update. J Am Coll Cardiol 2010;55(22): 2413-26. doi: 10.1016/j.jacc.2009.10.085.
26. Makkar RR, Fontana G, Jilaihawi H, Chakravarty T, Kofoed KF, De Backer O, et al. Possible subclinical leaflet thrombosis in bioprosthetic aortic valves. N Engl J Med 2015;373(21):2015-24. doi: 10.1056/NEJMoa1509233.

27. Chakravarty T, Sondergaard L, Friedman J, De Backer O, Berman D, Kofoed KF, et al. Subclinical leaflet thrombosis in surgical and transcatheter bioprosthetic aortic valves: an observational study. Lancet 2017;389(10087):2383-92. doi: 10.1016/S0140-6736(17)30757-2.

28. Bortolotti U, Milano A, Mossuto E, Mazzaro E, Thiene G, Casarotto D. Early and late outcome after re-operation for prosthetic valve dysfunction: analysis of 549 patients during a 26 -year period. J Heart Valve Dis 1994;3(1):81-7.

29. Vogt PR, Brunner-LaRocea HP, Sidler P, Zünd G, Truniger K, Lachat M, et al. Reoperative surgery for degenerated aortic bioprostheses: predictors for emergency surgery and reoperative mortality. Eur J Cardiothorac Surg 2000;17(2):134-9. doi: 10.1016/S1010-7940(99)00363-2.

30. Jamieson WRE, Burr LH, Miyagishima RT, Janusz MT, Fradet GJ, Ling H, et al. Re-operation for bioprosthetic aortic structural failure-risk assesment. Eur Cardiotorac Surg 2003;24(6):873-8. doi: 10.1016/S1010-7940(03)00566-9.

31. Gurvitch R, Cheung A, Ye J, Wood DA, Willson AB, Toggweiler S, et al. Transcatheter valve-in-valve implantation for failed surgical bioprosthetic valves. J Am Coll Cardiol 2011;58(21): 2196-209. doi: 10.1016/j.jacc.2011.09.009.

32. López-Otero D, Teles R, Gómez-Hospital JA, Balestrini CS, Romaguera R, Saaibi-Solano JF, et al. Implante percutáneo de válvula aórtica, seguridad y eficacia del tratamiento del homoinjerto aórtico disfuncionante. Rev Esp Cardiol 2012;65(4): 350-5. doi: 10.1016/j.recesp.2011.11.019.

33. Dvir D, Webb JG, Bleiziffer S, Pasic M, Waksman R, Kodali S, et al. Transcatheter aortic valve implantation in failed bioprosthetic surgical valves. JAMA 2014;312(2):162-70. doi: 10.1001/jama.2014.7246.

34. Emery RW, Krogh CC, Arom KV, Emery AM, Benyo-Albretch K, Joyce LD, et al. The St. Jude Medical cardiac valve prosthesis: a 25-year experience with single valve replacement. Ann Thorac Surg 2005;79(3):776-83. doi:10.1016/j.athoracsur.2004. 08.

35. Chikwe J, Filsoufi F, Carpentier AF. Prosthetic valve selection for middle-aged patients with aortic stenosis. Nat Rev Cardiol 2010;7(12):711-9. Online publication doi:10.1038/nrcardio.2010.164.

36. Bailey RD, Hart RG, Benavente O, Pearce LA. Recurrent brain hemorrhage is more frequent than ischemic stroke after intracranial hemorrhage. Neurology 2001;56(6):773-7. 
37. Niclauss L, von Segesser LK, Ferrari E. Aortic biological valve prosthesis in patients younger than 65 years of age: transition to a flexible age limit? Interact Cardiovasc Thorac Surg 2013;16(4):501-7. doi: 10.1093/icvts/ivs514.

38. Kaneko T, Cohn LH, Aranki SF. Tissue valve is the preferred option for patients aged 60 years and older? Circulation. 2013;128(12):1365-71. doi: 10.1161/CIRCULATIONAHA.113.002584.

39. Shazly A, Afifi A. RE-ALIGN: First trial of novel oral anticoagulant in patients with mechanical heart valves - The search continues. Global Cardiol Science and Pract 2014;2014(1):1-3. doi: $10.5339 / \mathrm{gcsp} .2014 .13$.

40. Puskas J, Gerdisch M, Nichols D, Quinn R, Anderson C, Rhenman B, et al. Reduced anticoagulation after mechanical aortic valve replacement: interim results from the prospective randomized On-X anticoagulation clinical trial randomized Food and Drug Administration investigational device exemption trial. J Thorac Cardiovasc Surg. 2014; 147(4):1202-11. doi: 10.1016/j.jtcvs.2014.01.004.

41. Oterhals K, Fridlund B, Nordrehaug JE, Haaverstad R, Norekval TM. Adapting to living with a mechanical aortic heart valve: a phenomenographic study. J Adv Nurs. 2013;69(9):2088-98. doi: $10.1111 / j a n .12076$.

42. Anyanwu A. Surgical aortic valve replacement: biologic valves are better even in the young patient. American College of Cardiology. 2015. Disponible en: http://www.acc.org/latest-in-cardiology/articles/ 2015/03/03/09/28/surgical-aortic-valve-replacementbiologic-valves-are-better-even-in- the-young-patient

43. Chan WS, Anand S, Ginsberg JS. Anticoagulation of pregnant women with mechanical heart valves: a systematic review of the literature. Arch Intern Med 2000;160(2):191-6.

44. McLintock C, McCowan LME, North RA. Maternal complications and pregnancy outcome in women with mechanical prosthetic heart valves treated with enoxaparin. J Obstet Gynaecol 2009;116(12): 1585-92. doi: 10.1111/j.1471-0528.2009.02299.x.

45. Hung L, Rahimtoola SH. Prosthetic heart valves and pregnancy. Circulation 2003;107(9):1240-6. doi: 10.1161/01.CIR.0000060806.86686.EC.

46. Briffa N, Chambers JB. Biological valves in younger patients undergoing aortic valve replacement. A word of caution. Circulation 2017(12);135:1101-3. doi: 10.1161/CIRCULATIONAHA.116.026385. 